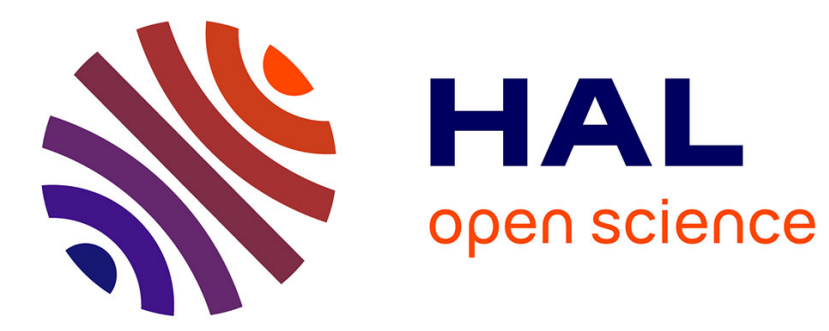

\title{
Témoignage et intériorité selon Kierkegaard
}

Emmanuel Housset

\section{To cite this version:}

Emmanuel Housset. Témoignage et intériorité selon Kierkegaard. Revue des sciences philosophiques et theologiques, 2002, Expérience chrétienne et philosophie: Kierkegaard, t. 86 (2), pp.205-228. 10.3917/rspt.862.0205 . hal-01870994

\section{HAL Id: hal-01870994}

\section{https://hal-normandie-univ.archives-ouvertes.fr/hal-01870994}

Submitted on 21 Sep 2018

HAL is a multi-disciplinary open access archive for the deposit and dissemination of scientific research documents, whether they are published or not. The documents may come from teaching and research institutions in France or abroad, or from public or private research centers.
L'archive ouverte pluridisciplinaire HAL, est destinée au dépôt et à la diffusion de documents scientifiques de niveau recherche, publiés ou non, émanant des établissements d'enseignement et de recherche français ou étrangers, des laboratoires publics ou privés. 


\section{Témoignage et intériorité selon Kierkegaard}

Emmanuel Housset

Le retour à soi se comprend souvent comme un projet de maitrise de soi et de transparence à soi rendant possible une appréhension objective du monde. Dans cette perspective l'opposition entre l'intériorité et l'extériorité se trouve dépassée parce que l'intériorité, sous la forme du je transcendantal, se comprend comme le lieu d'un pouvoir sur le monde : c'est elle qui constitue ce monde et ainsi fait de toute chose du monde un lieu où elle se retrouve finalement elle-même. Une telle conception de l'intériorité propre à l'idéalisme finit donc par retirer au monde tout caractère d'altérité puisque la chose se trouve réduite au statut d'un objet constitué par la conscience. En comprenant ainsi l'intériorité comme une pure immanence, comme uns sphère close sur elle-même dans laquelle l'extériorité n'est qu'une forme de l'intériorité, on est nécessairement conduit à faire du sujet qui contemple le monde un pur spectateur désintéressé de ce monde qui découvre son pouvoir de constitution et qui peut ainsi se considérer comme étant en quelque sorte propriétaire de la vérité qu'il donne à voir. Un tel spectateur transcendantal, qui se contemple en contemplant ce qu'il constitue, reçoit bien des données sensibles, mais il n'est, à proprement parler, touché par rien puisque rien ne peut le déloger de l'abri de sa transcendance par rapport à tout vécu. Il ne reçoit rien du dehors et tout ce qu'il est vient de ce qu'il se donne en se connaissant lui-même. Autrement dit, il ne se reçoit pas de ce qui lui advient et en conséquence il ne se donne jamais au point de mettre en péril la pure coïncidence avec soi qui le définit. Procédant ainsi par pure identité, un tel sujet ne peut devenir autre, il ne peut faire l'épreuve de sa propre altérité.

Or, une telle intériorité close dans laquelle l'altérité des choses, des autres hommes et finalement de soi-même se trouvent réduites est un enfermement en soi que Kierkegaard nomme le démoniaque. Contre une telle conception de l'intériorité, Kierkegaard va montrer que notre rapport à la vérité est tout autre : la vérité n'est pas ce que je produis, ni ce dont je serais le propriétaire ou le conservateur, mais elle est avant tout ce qui me touche et m'éclaire et en cela elle est le chemin et la vie du devenir soi. En prenant ainsi une signification existentielle, et non plus simplement propositionnelle comme la vérité d'un jugement, la vérité ne pourra plus être comprise comme étant relative à l'intériorité humaine, mais elle possédera un caractère essentiel d'altérité par lequel le je transcendantal sera destitué de sa toute puissance pour devenir plus humblement le «témoin de la vérité » qu'il a à être. On ne peut pas minimiser la profondeur du renversement effectué par Kierkegaard en substituant au moi qui se retrouve lui-même dans le monde le témoin qui accueille une vérité qu'il n'est pas. On ne peut donc comprendre ce que signifie témoigner de la vérité sans saisir en quoi l'existence est une épreuve de l'altérité qui est en quelque sorte toujours manquée dès que l'on fait du monde la seule altérité. Ainsi, Kierkegaard, en privilégiant l'étude du phénomène religieux, nous montre quelle est cette vérité qui nous donne de pouvoir parler en première personne dans le témoignage. En opposant le témoin au moi, Kierkegaard va montrer que l'individu ne peut accéder à sa vérité, à la singularité 
absolue de sa vocation, qu'à partir de l'épreuve de sa non-vérité et de son impuissance. Il montre du même coup indirectement l'impossibilité d'expliquer en quoi le témoignage n'est pas la simple transmission d'une connaissance sans enraciner cette pensée du témoignage dans une compréhension du sens original de la vérité. Le témoin n'est-il qu'un autre nom du je transcendantal en ce qu'il se constitue lui-même comme témoin et fait du jugement le lieu de la vérité ou bien est-ce la vérité elle-même qui, en se manifestant, fait de moi un témoin en requérant absolument ma parole? N'est-ce pas dans ce second cas que le témoignage n'est pas une simple transmission, mais un acte qui met radicalement en question celui qui témoigne parce qu'il l'engage tout entier?

\section{L'excès du soi}

L'intériorité ne se laisse pas définir pour Kierkegaard par la seule réflexivité qui fonde le pouvoir sur soi et sur le monde. En effet, si l'intériorité est avant tout réflexivité alors le mode d'être de l'homme est nécessairement autarcique : être soi c'est penser et agir par raison pure, c'est-à-dire obéir à la loi que l'on trouve en soi, et cette conscience dédoublée prend ainsi la forme d'un tribunal intérieur. Or, pour Kierkegaard, antérieurement à cette réflexivité il y a une épreuve de sa non-vérité qui la rend possible et qui la reconduit à son véritable telos. En inversant le principe de la subjectivité, Kierkegaard donne à voir en quoi l'homme ne se saisit lui-même dans toutes ses dimensions et dans la singularité absolue de son être que par la conscience de sa finitude et de sa faute puisque la crainte et le tremblement reconduisent à soi plus radicalement et plus sûrement que toute réflexion. Dès lors, pour éviter le mode d'être autarcique de l'homme s'agit-il seulement de reconnaître que la vérité dont l'homme témoigne est nécessairement une vérité qui se révèle historiquement? Pour Hegel c'était bien la reconnaissance de ce caractère historique de la vérité qui ouvrait une place pour le témoignage dans la connaissance. Or, si pour Kierkegaard également témoigner ce sera rapporter comment la vérité s'est manifestée dans l'histoire, il s'opposera à Hegel en refusant l'idée que le témoignage puisse être réduit par le savoir absolu. Si la vérité n'est pas d'abord ce qui se prouve par réflexion, mais ce qui s'éprouve, il est impossible d'en faire un chez soi et le témoignage marquera justement l'altérité irréductible de la vérité qui s'est manifestée dans l'histoire et pas seulement sa préalable extériorité au soi dans le temps. On comprend alors que pour Kierkegaard la question directrice n'est pas de savoir comment à partir de mon intériorité il m'est possible d'atteindre une extériorité ou comment je peux sortir de moi-même pour atteindre un objet et donc pour accéder à l'objectivité. La question essentielle pour lui n'est pas de résoudre le problème de la connaissance transcendante, ni même de prendre l'objet comme fil conducteur pour l'étude de la vie intérieure, mais elle est pour lui de comprendre véritablement pour elle-même le sens original de l'expérience intérieure. Si Kierkegaard est le penseur de l'intériorité c'est que plus que tout autre il a fait vraiment de l'intériorité la question directrice et non ce qui intervient pour résoudre une autre difficulté.

La thèse centrale du Post-scriptum selon laquelle «la subjectivité, l'intériorité est la vérité » a pour but de dire l'existence comme intériorité en marquant l'essentiel devenir de l'intériorité. Cependant, pour que l'intériorité soit la vérité, elle doit d'abord prendre conscience de sa non-vérité c'est-à-dire saisir qu'elle n'est pas d'emblée dans la vérité parce que la vérité ne vient pas d'elle mais lui est donnée. Plus précisément, la conscience de soi n'est pas seulement le savoir initial que je ne me connais pas moi-même et que je dois apprendre à me connaittre, mais elle est la conscience que je ne suis pas à moi-même mon propre maitre et que cette non maîtrise, cette non-vérité, n'est pas seulement justement initiale, comme si elle pouvait être surmontée par un acte de réflexion. Une telle non-vérité est continue et c'est donc en un tout autre sens que l'on n'en a jamais fini de devenir soimême : ce n'est pas que le travail de surmonter sa finitude soit infini qui justifie ici l'écart de soi à soi, mais l’idée que plus on est témoin de la vérité plus on prend conscience de sa 
finitude. Dans cette perspective la connaissance de soi conduit à comprendre que le principe de mon être, de mon unité, ne vient pas de moi et qu'il ne s'agit plus de rechercher la transparence à soi nécessaire pour la maîtrise, mais d'être au-delà de soi, autrement dit d'exister. En conséquence, le devenir soi ne peut plus être compris comme le déploiement progressif d'une essence par le pouvoir de la réflexion. Il s'agit pour Kierkegaard d'un devenir autre radical devant la transcendance de Dieu, qui seul permet d'éviter le démoniaque, c'est-à-dire le mouvement de se cacher soi-même, qui est l'échec de la communication et donc l'échec le plus grave de la vie intérieure comme vie qui devrait être un accès à l'éternel en soi. En effet, un tel mouvement d'intériorisation de sa relation à l'absolu, à l'éternel, est impossible dans un acte d'affirmation de soi et il suppose au contraire un acte d'humilité qui consiste à avoir renoncé à l'autonomie de la subjectivité. Si, comme le dit Le concept d'angoisse, «la vie intérieure est l'éternité ou la détermination de l'éternel en l'homme ${ }^{1}$, elle ne consiste pas ici dans l'accomplissement d'une pleine présence à soi, mais dans une reconnaissance d'une absence d'accès direct à la vérité à partir de la subjectivité : je ne possède pas en moi les moyens d'accéder à la vérité et cela marque la radicale extériorité de ce qu'il s'agit d'intérioriser. En ce sens, l'accroissement de l'intériorité quand on passe de l'intériorité immédiate du sentiment à l'intériorité éthique puis à l'intériorité de la foi est en même temps un accroissement de l'épreuve de l'altérité. Dès lors, dans un tel accroissement il n'est pas question de déterminer sa vie selon des normes universelles, mais de découvrir en soi l'absolu qui fonde notre existence et qui tout à la fois nous excède et nous donne d'être. Un tel excès de ce que l'on est sur ce que l'on pense être ou pouvoir être est ce qui ne peut entrer dans aucun système de l'existence, dans aucune conception du sujet pur pleinement présent à lui-même telle qu'elle a pu être formulée par Hegel. Ainsi, cet excès du soi montre qu'exister ne consiste pas à se retrouver partout dans le monde pour être en tout lieu chez soi, ne consiste pas à vivre dans la certitude d'être toute réalité, c'est-à-dire dans la certitude que l'extérieur n'est que l'empreinte objective de l'intérieur. En effet, il ne s'agit pas tant de résoudre le conflit de l'individu avec le monde en réduisant l'altérité de l'objet, mais bien d'exister en se recevant de l'épreuve d'une altérité irréductible, c'est-à-dire de s'arracher à cette crise beaucoup plus grave qu'est la fermeture sur soi du démoniaque en étant un simple témoin de la vérité.

\section{Témoignage et communication indirecte}

Il appartient à l'essence de l'intériorité de se communiquer, mais toute communication n'est pas pour autant un témoignage et c'est pourquoi Kierkegaard distingue la communication inter-individuelle ordinaire, qui est tout immédiate, directe de sujet à sujet, et la communication indirecte et seule véritable qui est celle de l'intériorité. En effet, de même qu'il ne peut pas y avoir une appropriation directe des normes générales, parce qu'elles demeureraient parfaitement abstraites, mais seulement une patiente intériorisation par laquelle on les devient, il n'y a pas non plus de transmission directe du savoir à autrui dans le domaine des vérités éthico-religieuses. Personne dans ce domaine, mais cela vaut aussi des autres domaines, n'est maître de la vérité et c'est pourquoi «le secret de la communication consiste à libérer l'autre $»^{2}$. En effet, toute communication directe de l'intériorité serait un sacrilège dans la mesure où la communication éthicoreligieuse n'est pas tant celle d'un savoir que celle d'un pouvoir-devoir. En cela elle a un sens d'abord existentiel et ne peut être qu'inconditionnellement indirecte ${ }^{3}$. Il n'y a dans cette communication aucun anonymat possible, pas même celui du «nous les philosophes »,

\footnotetext{
1 VII, 246-247. Les textes de Kierkegaard seront cités d'après la traduction française des CEuvres complètes par P.-H. Tisseau et E.-M. Jacquet-Tisseau en vingt volumes, Paris, Editions de l'Orante, 1966-1986. On indiquera désormais uniquement le numéro du volume et la page.

2 Post-scriptum définitif et non scientifique aux Miettes philosophiques, X, p.71.

${ }^{3}$ Cf. La dialectique de la communication éthique et éthico-religieuse, XIV, p.374.
} 
puisque toute parole vient d'un individu qui s'expose en personne pour s'adresser à un individu : "La vérité éthico-religieuse se rapporte essentiellement à la personnalité ; elle ne peut être transmise que par un 'je' à un autre 'je'»". Il est clair qu'ici le «je » n'est pas le «je pur et ventriloque fruit de mon imagination $»^{5}$, qui n'est en fait le je de personne, mais est le je qui parle en première personne et qui ne se contente donc pas de transmettre la vérité, mais qui dit cette vérité éthique en la devenant. Ainsi, après avoir souligné l'inhumanité de notre époque qui n'a jamais autant communiqué directement et pourtant dans laquelle les individus ne se disent plus rien, Kierkegaard rappelle la nécessité d'une parole en première personne pour inciter l'autre à effectuer la double réflexion de la pensée. Dès lors, la vérité éthico-religieuse ne peut être communiquée que par un individu qui la vit et en reçoit en retour le titre de personne. Certes, la finitude de l'homme fait que sa communication ne peut pas être pleinement indirecte, mais il n'en demeure pas moins que la communication d'un devoir doit être la plus indirecte possible et que tout ce qui reste de communication directe éloigne de la vérité éthique en étant un obstacle à son appropriation par un autre homme. Ainsi, paradoxalement, quand la vérité prend une signification existentielle, ce n'est plus l'impersonnel qui est la condition de son intériorisation: plus je deviens une vérité éthique, plus j'aide l'autre à la devenir. L'impersonnalité, l'anonymat d'un je pur, devient ici ce qui fait obstacle à l'intériorisation de la vérité par quelqu'un d'autre. Il est donc essentiel de ne pas tout réduire à la communication directe qui, comme pur rapport d'esprit à esprit, est transparente, et de reconnaitre la place de la communication indirecte qui est elle un rapport d'individu à individu et qui n'a donc lieu que sous le sceau du secret de chaque intériorité. En effet, un tel travail d'intériorisation propre à un individu est toujours incomparable et le secret manifeste contre toute pensée abstraite qu'il s'agit bien d'être cette vérité et non pas de «cesser d'être homme pour devenir un livre »'.

En montrant ainsi que toute connaissance qui ne se tourne pas vers l'intérieur est contingente $^{7}$, Kierkegaard marque son refus de la pensée spéculative qui en oubliant la personne oublie aussi l'existence et enferme dans la seule contemplation de soi, c'est-à-dire dans la contemplation du vide : le je pur est un je du vide plus radicalement anonyme que le on heideggerien ${ }^{8}$. Alors que la connaissance de soi des philosophes conduit finalement à n'être que le spectateur de soi, le spectateur d'un soi auquel rien n'arrive et qui n'est que le vide de la coïncidence avec soi, le vrai témoin de la vérité ce n'est jamais sur lui qu'il porte témoignage et dans cette perspective, pour le christianisme, le témoin de la vérité n'est pas d'abord celui qui raisonne ou qui doute, mais il est celui qui obéit et qui renonce à ériger la sagesse humaine en Providence ${ }^{9}$. En conséquence, le témoignage, qui se trouve nécessairement disqualifié dans la communication directe du savoir, reçoit une place essentielle dans la communication indirecte du pouvoir parce que l'intériorité a à transmettre une vérité qu'elle ne produit pas. Certes, seul le Christ est la forme achevée de la communication indirecte et en cela il est le "témoin fidèle », selon l'expression d'Apocalypse 1,5, mais le témoignage humain demeure une communication à la fois directe et indirecte qui incite l'homme à se décider. En effet, le témoignage est une communication directe dans la mesure où il s'agit de parler de soi devant ses semblables, mais il est en même temps communication indirecte du fait qu'il s'adresse d'abord à Dieu qui seul peut en être le juge : «Le 'témoignage' est au vrai la forme de message exactement équidistante du message directe et du message indirect. Le témoignage est bien communication directe, mais sans faire cependant des contemporains l'instance. Car tandis qu'il adresse son

\footnotetext{
${ }^{4}$ Ibidem, p.375-376.

${ }^{5}$ Ibidem, p.376.

${ }^{6}$ Post-scriptum, X, p.88.

${ }^{7}$ Cf. Post-scriptum, X, p.184.

${ }^{8}$ Cf. Jean-Louis Chrétien, La voix nue, ch.8, Minuit, 1990.

${ }^{9}$ Cf. Dialectique de la communication, XIV, p.370-371.
} 
message aux contemporains, le témoin s'adresse à Dieu et fait de lui l'instance $»^{10}$. N'étant ni un simple spectateur, ni le Christ, l'individu humain ne peut dissocier en lui la transmission directe et la communication indirecte et c'est en ce sens là qu'il est au sens propre un témoin, c'est-à-dire quelqu'un qui ne se contente pas de savoir mais qui veut être cette vérité sans pouvoir jamais s'identifier pleinement à cette vérité, sans jamais pouvoir dire «je suis la vérité ».

Qui peut-il se dire lui-même témoin de la vérité ? Ne serait-ce pas témérité ? Quoi qu'il en soit pour l'instant de cette difficulté, il est clair que le témoignage n'est pas un simple discours direct et qu'en cela ce n'est pas la façon dont il est reçu par les autres hommes qui en atteste la vérité. Mais en retour la seule communication indirecte n'est pas non plus un témoignage car il ne s'agit pas seulement d'être cette vérité, mais bien aussi de la dire. Ainsi, témoigner de la vérité est bien une communication directe-indirecte qui consiste à être devant Dieu tout en s'adressant à ses contemporains : il s'agit de dire la vérité aux autres tout en l'existant ${ }^{11}$. Ni l'autre homme, ni moi-même nous ne sommes la mesure de la vérité éthique que je transmets et c'est devant le crucifié et lui seul que ma parole est jugée. Ainsi, «la première condition absolue de toute communication de la vérité est la personnalité ${ }^{12}$, mais si le témoin parle en première personne, il n'est pas la mesure de la vérité et il reçoit le pouvoir de parler de la vérité elle-même. Cependant, si témoigner consiste bien à dire et à se dire, il ne s'agit pas tant de parler de soi que de dire le paradoxe en soi, de se dire comme paradoxe, c'est-à-dire comme personne : témoigner consiste à dire la vérité qui me donne d'être et qui m'éclaire. En cela le témoin est le contraire d'un simple spectateur de la vérité puisqu'il ne se contente pas de parler de la vérité, mais il parle dans la vérité, à partir d'elle. Le témoignage est donc tout autre chose qu'une simple occupation extérieure, qu'une simple conduite quotidienne: il est un événement fondamental de l'existant. En effet, le témoignage est une structure de l'existence qui consiste à accomplir sa vocation dans la mesure où il est une réponse à l'assignation à témoigner qui vient de l'absolu lui-même. Heidegger reprendra sous une autre forme cette idée que le témoignage est l'acte de se donner tout entier en répondant à l'appel de ce que l'on a à être : "Qui est donc l'être humain? Celui qui doit témoigner ce qu'il est. Témoigner signifie d'une part révéler, dénoncer; mais en même temps cela veut dire aussi: répondre, dans la dénonciation, de ce qui est dénoncé. L’homme est celui qu'il est, précisément dans l'attestation de son être-là propre $»^{13}$. Certes, pour Heidegger ce que l'homme doit attester est son appartenance à la terre et non son obéissance à Dieu, mais dans les deux cas le témoignage de ce qui lui donne d'être est ce qui constitue son être-homme et non ce qui intervient après coup. En cela la communication éthique n'a pas vraiment d'objet, mais elle consiste à s'exposer dans son être propre : exister, c'est témoigner, c'est-à-dire marquer sa dette infinie vis-à-vis de ce qui nous advient, puisque ce qui se donne à moi, en me donnant la possibilité de devenir moi-même dans la réponse, me donne plus que ce que je pourrais jamais rendre.

\section{La passion de la vérité}

On ne peut déterminer ce qu'il en est d'exister sans comprendre notre essentielle passion de la vérité : «le plus haut degré de l'intériorité chez un sujet existant c'est la

\footnotetext{
10 Journal X1 A235, trad. franç. K. Ferlov et J.-J. Gateau, 5 volumes, Paris, Gallimard, 1941-1961, tome 3, p.82.

${ }^{11}$ Cf. P. Jacquemont, J.-P. Jossua, B. Quelquejeu, Le temps de la patience, Etude sur le témoignage, Cerf, 1976.

12 Journal, X1 A531, tome 3, p.160.

13 "Wer ist der mensch? Jener, der zengen muss, was er sei. Zeugen bedeutet einmal ein Bekunden; aber zugleich meint es : für das Bekundete in der Bekundung einstehen. Der Mensch ist der, der er ist, eben in der Bezengung des eigenen Daseins ", Heidegger, Hölderlin und das Wesen der Dichtung, G.A. tome 4, Klostermann, 1981, p.36, trad. franç. Approche de Hölderlin, Gallimard, 1973, p.45.
} 
passion, passion à laquelle correspond la vérité en tant que paradoxe $»^{14}$. Cette thèse qui contient le cœur de la pensée de Kierkegaard souligne bien que la vérité est ce dont je témoigne et non ce que je produis dans la mesure où cette passion éthique, et non esthétique, est une radicale transformation de mon existence qui crée en moi un rapport existentiel à l'éternel. Dans une telle passion la vérité ne peut devenir qu'un paradoxe et l'homme lui-même devient un paradoxe puisqu'en recevant la vérité il porte en lui une différence irréductible. En effet, la vérité est un paradoxe parce qu'elle ne m'appartient pas et l'homme est un paradoxe parce qu'il porte en lui le rapport entre deux réalités incommensurables : la vérité impersonnelle et objective et la subjectivité comme passion d'un sujet particulier. Dans cette perspective, la personne, qui n'est pas ici l'exemplaire d'une espèce, ni seulement l'individu qui tente de se différencier de l'espèce, est donc un paradoxe absolu parce qu'elle ne peut être vraie de par sa propre vérité et parce que la vérité ne peut se définir comme l'accord de soi avec soi. Autrement dit, il n'y a pas de commune mesure entre l'homme et cette vérité qui ne lui appartient pas et pourtant il ne peut lui-même devenir vrai que par cette vérité à laquelle il ne peut appartenir. L'homme n'est pas à lui-même son propre principe, il n'est pas à lui-même son propre maître, et quand il cherche malgré tout à s'absolutiser il ne peut que tomber dans le désespoir.

Or, si l'existence éthique est paradoxe parce qu'elle consiste à se déterminer selon un telos qui ne se trouve ni en nous, ni dans le monde fini, cette possibilité d'appartenir à la vérité malgré tout suppose pour le sujet de faire l'épreuve de sa non-vérité, de son éloignement de la vérité que Kierkegaard nomme dans le Post-scriptum le péché : «Appelons péché la non-vérité de l'individu »" La conscience de son péché n'est pas la simple conscience de la faute dans la mesure où elle n'est pas la transgression d'une loi morale, mais un refus de la vérité et c'est pourquoi elle est profondément singularisante. Le péché étant radicalement mien parce qu'il n'est pas une absence, une faiblesse, mais un refus, la conscience du péché sauve de l'impersonnel, de l'anonymat de la foule qui n'a pas de mains. Ce n'est donc que la conscience de son péché qui permet de résister à la tentation d'une fuite dans l'anonymat qui accompagne constamment le devenir soi : on n'en a jamais fini avec sa non-vérité et c'est là que naît la conscience de sa vérité ${ }^{16}$. En effet, le péché est une obscurité à soi plus radicale que la simple ignorance et c'est pourquoi l'intériorité ne peut être la vérité qu'à partir de cette conscience de sa non-vérité. Cette obscurité est liée à un refus de soi, fut-ce dans l'affirmation de soi comprise dans l'idéal d'une maittrise de soi sans reste, puisqu'il consiste à ne pas accepter de se recevoir de ce qui nous est donné. Ainsi, prendre conscience d'un tel refus c'est aussi prendre conscience de l'impossibilité de se fonder soi-même et c'est cela qui ouvre à son véritable telos. En effet, dans cette conscience l'intériorité est existence et la vérité demeure un paradoxe. Plus précisément, le péché marque un éloignement vis-à-vis de la vérité, et une absence de communauté d'essence entre l'homme et la vérité, beaucoup plus forte que la simple ignorance, et de ce fait l'accomplissement de soi n'est pas le même à partir du péché et à partir de l'ignorance : l'obscurité du péché fait qu'on n'en aura jamais fini de devenir soi-même parce qu'on n'en aura jamais fini avec sa non-vérité. Cela signifie positivement que l'on ne peut devenir soi que dans la conscience de n'être pas soi et dans la conscience de l'impossibilité de pouvoir jamais l'être totalement. La conscience de sa non-vérité est donc la condition première de l'appropriation de la vérité et elle est ainsi également la condition première de l'intériorité comme témoignage.

A partir de ce concept existentiel de vérité, la communication peut bien être comprise comme un rapport d'intériorité à intériorité : «Son intériorité, c'est le respect qu’il

\footnotetext{
14 Post-scriptum, X, p.185.

${ }^{15}$ X, p.194.

16 Cf. Jean-Louis Chrétien, La voix nue, Minuit, 1990, p.164 : «La non-vérité est l'index existentiel de la vérité ».
} 
porte au disciple et à l'intériorité qu'il est ${ }^{17}$. En effet, le respect de l'activité «personnelle » de l'autre fait qu'il s'éloigne de moi et cela fonde justement la relation interpersonnelle. Kierkegaard montre en quoi l'intériorité du maître consiste à témoigner d'une vérité éthique dont il n'est pas la mesure et qui l'excède. En conséquence, si le témoin n'est pas le propriétaire de la vérité, il ne peut pas être non plus celui qui en assure la conservation puisqu'il est lui-même celui qui a été saisi par la vérité dans son être propre, c'est-à-dire encore une fois dans son péché. La vérité éthique dont il s'agit ici, et qui est d'une toute autre nature que la vérité propositionnelle comme «le chat est noir», n'est pas une vérité dont on exhibe la preuve, mais une vérité dont on fait l'épreuve et c'est justement de cette épreuve dont on témoigne. En cela, c'est bien parce qu'il s'agit de transmettre non pas un savoir mais une épreuve que cette communication est l'origine de la personnalité comme être saisi par la vérité. Même si je sais que je n'en aurais jamais fini avec la tentation de vouloir faire mienne une vérité que je sais n'être pas à moi, je ne m'arrache à l'anonymat et je ne deviens moi-même que dans l'éloquence du témoignage et de ce fait la vérité que je montre par mon témoignage me montre également comme esprit advenant à lui-même, comme esprit en souffrance de lui-même. Finalement, en montrant comment l'anonymat premier, la fuite dans l'impersonnel, offre à la personnalité son espace de révélation, Kierkegaard décrit également ce qu'il en coûte de témoigner, ce qu'il en coûte d'être soi. De ce point de vue, seul le témoignage montre véritablement ce que peut l'homme et ne retire pas à l'autre homme le pouvoir de témoigner, mais au contraire l'éloigne de soi pour mieux le reconduire à la solitude de son travail d'intériorisation. Le maittre n'est qu'un témoin de l'esprit qui, en disant la vérité qui l'éclaire, libère l'autre en le laissant être esprit, c'est-à-dire en lui montrant la nécessité d'avoir de la personnalité, c'est-à-dire d'avoir le courage d'affirmer sa conviction. Ainsi, le témoignage est une mobilisation de l'existence tout entière, qui souvent suscite la violence des autres hommes du fait même qu'en lui on n'avance pas masqué, mais qu'on se présente en personne, nu. Le témoin de la vérité, dans sa nudité essentielle, suscite cette violence étonnante, qui s'exerce envers ceux qui disent la vérité uniquement parce qu'ils la disent, dans la mesure où il laisse se manifester l'éclat de cette vérité qui me montre dans ma fermeture sur moi-même. On peut donc dire que c'est toujours de la vérité dont on témoigne, au risque de sa vie, et qu'en cela le témoignage est l'accomplissement de sa vocation : se donner tout entier à la manifestation de la vérité est ce qui peut seul briser en l'autre la fermeture à l'accueil de la vérité. Face à un témoin de la vérité il n'y a qu'une seule alternative : ou la haine ou l'amour. De ce fait, le témoin de la vérité n'est pas seulement celui qui dit quelque chose de vrai : "'Témoin de la vérité'. Par ce mot, on ne désigne certainement pas quiconque dit quelque chose de vrai ; sinon, Dieu merci, nous ne manquerions pas de 'témoins de la vérité'. Non, quand on parle de 'témoin de la vérité', il faut entendre que l'existence personnelle sur le plan éthique est conforme à ce que l'on dit et exprime $»^{18}$. En effet, le témoin de la vérité existe cette vérité et donc il ne peut agir autrement ${ }^{19}$. Ainsi, en dépassant d'une toute autre façon que Hegel l'opposition simple entre l'extériorité et l'intériorité, Kierkegaard montre que l'intériorité authentique consiste à agir dans la conscience de sa responsabilité éternelle, c'est-à-dire non pas à marquer le monde de mon empreinte, mais à être là où la vérité m'appelle.

En témoignant de la vérité, notre existence tout entière devient ainsi une tâche, la tâche ardue de devenir soi qui ne consiste pas à parvenir à son identité, à sa vérité, mais à recevoir de la vérité elle-même son identité. En cela l'intériorité ne peut jamais être comprise comme la pure contemplation insouciante de soi, mais comme une épreuve de la vérité qui arrache à cette douillette intimité pour assigner à agir et qui du même coup me dévoile une toute autre ipséité. Cela dit, cette révélation de la vérité, qui m'assigne à dire et

\footnotetext{
${ }^{17}$ Post-scriptum, X, p.224.

18 Point de vue explicatif de mon cuvre, XVI, p.95.

${ }^{19}$ Cf. Deux petits traités éthico-religieux, XVI, p.128.
} 
à être, c'est-à-dire à témoigner, n'a rien d'une extériorité tapageuse qui fait d'autant plus de bruit qu'elle masque le vide du soi, mais elle est plutôt une extériorité discrète, celle des grandes décisions intérieures ${ }^{20}$. La vérité est toujours ce qui à la fois veille et réveille le dormeur que je suis pour qu'en témoignant d'elle je tente de porter ce rapport absolu au telos absolu, tout en assumant mon rapport au monde, c'est-à-dire mon telos relatif. Or justement, vivre ainsi en témoin de la vérité dans le monde fini, sans mettre sa vie dans ce monde, est une souffrance essentielle à l'intériorité : «L'intériorité (l'individu éthique et éthique-religieux) voit dans la souffrance l'essentiel $»^{21}$. Cette souffrance particulière est celle liée à la tâche de réaliser l'éternel dans le temporel et de ce point de vue «l'amour humain est l'invention la plus belle, bien que fragile, de la temporalité $»^{22}$. Le témoin est donc celui qui met sa vie dans cette passion de la vérité, dans ce pâtir essentiel et non accidentel dans lequel le moi se livre lui-même à la contrainte de la vérité : «L'humain, c'est l'intériorité cachée dans la passion absolue $»^{23}$. Le témoin, en se liant ainsi à ce qui le libère, peut faire face aux moqueries du monde, peut ne pas céder à la tentation d'une fuite dans l'impersonnel issue de l'épreuve du monde. Alors que l'épreuve du monde est dépersonnalisante, l'épreuve de la vérité est essentiellement personnalisante et donc seul le témoin est vraiment une personne.

\section{Le témoignage chrétien}

Tout ce qui précède conduit à montrer que la vraie signification du témoignage se révèle pleinement pour Kierkegaard dans le témoignage chrétien car la religion, plus que l'éthique, consacre cet être saisi par la vérité. En effet, l'éthique comme accomplissement du devoir demeure un témoignage de son être-là propre, de l'affirmation de soi dans le choix, plus qu'un témoignage de la vérité qui comme vérité du moi suppose son impuissance radicale. Dans cette perspective on pourra contester la légitimité d'une proposition comme «je suis témoin » parce que ce n'est pas le «je » qui témoigne mais bien la personne. Paul Ricoeur peut bien soutenir que «c'est le témoin qui d'abord se déclare témoin $»^{24}$, on peut légitimement s'interroger sur les conditions de possibilité d'une telle auto-désignation, d'une telle auto-constitution du témoin qui ne serait que confirmée par celui qui reçoit le témoignage, et cela même dans le seul cadre du savoir historique puisque la perspective épistémologique n'est jamais totalement séparable d'une perspective ontologique. En effet, dans une telle thèse, la présence d'une structure dialogale ne change rien à la nature du témoignage. Certes, même dans le concept biblique (et pas seulement juridique) de témoin ( $\mu \alpha \rho \tau \cup \varsigma)$, le témoin est celui qui atteste de ce qu'il a vu et celui qui est cru sur parole. Dieu est ainsi pour la Bible le témoin véridique de l'intériorité humaine. En ce qui concerne le témoin humain, qui n'est pas nécessairement oculaire, il proclame ce qu'il sait et ainsi, tel l'apôtre, il rend témoignage devant les hommes. En cela toute vocation est un $\mu \alpha \rho \tau u \rho t o v$ qui dit au monde l'événement du salut. Le témoignage est donc une confession, une louange à Dieu, et de ce point de vue il ne peut jamais se réduire à une simple transmission persuasive. Il ne s'agit pas en effet de mettre le ton de la force au service de la persuasion, mais au contraire de laisser la vérité s'attester elle-même à travers $\mathrm{moi}^{25}$. Dès lors, si l'engagement du témoin semble bien être l’invariant des différentes

\footnotetext{
${ }^{20}$ Cf. Post-scriptum, XI, p.79.

21 Post-scriptum, XI, p.126.

22 Les cuvres de l'amour, XIV, p.288.

${ }^{23}$ Post-scriptum, XI, p.196.

${ }^{24}$ La mémoire, l'histoire, l'oubli, Seuil, 2000, p.204.

${ }^{25}$ Cf. Ceslas Spiq o. p., Lexique théologique du Nouveau Testament, Cerf-éditions universitaires de Fribourg, 1991, art. $\mu \alpha \rho \tau u \varsigma$, p.969-974. Sur ce même terme on consultera aussi utilement le Theologisches Wörterbuch zum Neuen Testament, tome 4.
} 
significations du témoignage $e^{26}$, il semble cependant difficile de faire pour autant de l'appropriation du témoignage, par laquelle je le dis mien, un caractère originaire du témoignage. Autrement dit, il faut reconnaitre malgré tout, quand on passe au concept chrétien du témoignage, une rupture de sens puisque l'engagement du témoin dans le témoignage ne résulte plus ici d'une décision personnelle du témoin qui se donne ses propres possibilités, mais d'une décision libre de dieu qui cette fois ouvre mes propres possibilités. Certes, le témoin fidèle demeure celui qui agit, mais dans la mesure où il s'abandonne à la vérité dont il témoigne, il ne peut plus parler de «son » témoignage. Ainsi, il est sans doute possible de contester sur ce point la thèse de Paul Ricoeur selon laquelle le sens prophétique du témoignage vient seulement s'ajouter au sens profane, sédimenté, sans le modifier. N'est-ce pas forcer la continuité du sens que de vouloir montrer que le sens biblique du témoignage intègre le sens d'engagement, le sens juridique du procès et le sens empirique de récit des choses vues? Il est clair que pour une herméneutique du témoignage une telle perspective permet d'éviter la pure et simple homonymie, mais n'interdit-elle pas aussi en retour l'élucidation d'une véritable généalogie du sens? L'herméneutique du témoignage de Paul Ricoeur demeure liée à la conception traditionnelle de la vérité et c'est pourquoi elle montre la nécessité de transformer le témoignage en objet d'interprétation. Dès lors, même si au départ le témoin n'est pas propriétaire de son témoignage, il doit bien ensuite se transformer en observateur critique et ainsi s'approprier «son » témoignage. La question n'est pas alors seulement de savoir si cela respecte la dimension chrétienne du témoignage, mais elle consiste aussi à se demander si cette détermination du témoignage comme engagement d'un sujet qui constitue un objet respecte l'essence du témoignage en tant que tel. En effet, avec Kierkegaard, qui est particulièrement attentif à la discontinuité, on peut s'interroger sur le caractère inappropriable du témoignage chrétien qui semble être un engagement absolu, un abandon de soi. Un tel abandon n'est-il pas alors aussi la vérité de tout témoignage par rapport à laquelle la constitution de soi comme spectateur critique semble résulter d'un processus de «désengagement»? En cela il est peut-être possible d'établir que les autres significations du témoignage, liées à la détermination du témoin comme sujet épistémologique, ne sont pas en continuité mais bien dérivées, par abstraction, vis-à-vis de la signification chrétienne du témoignage. L'alternative est simple : ou le témoignage chrétien se réduit à n'être qu'une réalisation particulière de l'essence générale du témoignage, qui peut être saisie hors de tout contexte biblique, ou le témoignage chrétien, seul, manifeste véritablement le saut qui constitue l'essence du témoignage et alors il n'y a pas d'intelligence possible du témoignage sans une compréhension du saut de la foi.

Qu'est-ce qui fait alors de moi un témoin? Saint Augustin, notamment, donne à voir assez clairement la dimension chrétienne du témoignage en décrivant la transformation de Pierre en témoin : Pierre est d'abord celui qui malgré sa promesse ne témoigne pas, mais qui par crainte servile des moqueries du monde fuit dans l'anonymat. Cette figure de Pierre, qui a renié trois fois le Christ en une même nuit, est la figure même du chrétien et elle indique que la force, le courage et la fidélité du témoignage ne peuvent venir que de l'Esprit et non de soi parce que seul l'Esprit peut fortifier et dilater l'amour de la vérité. Ainsi, celui qui était tombé dans la crainte de l'esclave est devenu par la puissance de l'Esprit à la Pentecôte le vrai témoin qui a force et assurance et qui prêche le Christ sans crainte de la mort. C'est l'Esprit qui rend ses témoins inébranlables et qui leur permet de renverser l'incrédulité à la foi, la fermeture à la charité et le refus de la paix : «L'esprit en effet, en rendant témoignage au Christ et en rendant ses témoins inébranlables, a enlevé la crainte

${ }^{26}$ Cf. Paul Ricoeur, «l'engagement du témoin dans le témoignage est le point fixe autour duquel pivote l'éventail du sens », Lectures 3, Paris, Ed. du Seuil, 1994, p.117. 
aux amis du Christ et a changé en amour la haine de ses ennemis $»^{27}$. Le Christ fait de ses disciples ses témoins par l'Esprit qui rend notre parole éloquente, qui donne le pouvoir de convaincre et de résister aux persécutions. Ainsi, le témoin est ici celui qui enseigne à l'humanité tout ce que Jésus était et tout ce qu'il a prescrit (cf. Mt 28,19) et cette mission personnelle ne peut avoir lieu que dans l'amour ecclésial de la démesure de l'amour du Père. Dès lors, si le Christ est le «témoin fidèle », l'homme lui atteste que le Christ est Dieu et que sa vie tout entière s'identifie à son témoignage. Dans le Christ il y a identité entre le je et la mission, ce qui n'est pas le cas pour l'homme dont le témoignage reconduit au témoignage du Christ et s'accomplit nécessairement collectivement. Ainsi, le témoin engage sa vie entière dans une obéissance à la vérité, il donne sa vie pour la véritée ${ }^{8}$ et de cette façon il se révèle en révélant celui qui fait être. Cette révélation de soi n'est autre que l'accomplissement de l'amour inter-individuel auquel m'assigne l'amour de Dieu.

Cette brève mise en perspective permet de montrer que pour Kierkegaard il appartient à l'essence de tout témoin de ne pas se constituer lui-même comme témoin. En premier lieu le témoin de la vérité est le héros de la foi qui pour le bien qu'il fait reçoit en retour la haine de ses contemporains. Quand le Christ appelle dans le Nouveau Testament les disciples et les apôtres des témoins, «il s'agit d'hommes qui, dans un renoncement total, dans la pauvreté, l'humiliation, et ainsi prêts à toutes les souffrances ont pour mission d'aller dans le monde, ennemi mortel de la condition chrétienne dont il est l'expression contraire $»^{29}$. Cela signifie d'abord que «pour le christianisme, la vérité ne consiste pas à la savoir, mais à l'être $»^{30}$ et c'est pourquoi le vrai prédicateur, même si les hommes ne l'écoutent pas, se doit d'être lui-même, véridique devant Dieu, et ne doit pas s'oublier en figurant un personnage. En effet, l'homme qui se contente de dire une vérité sans l'être se réduit au regard vide d'un masque, c'est-à-dire se réduit à la pure possibilité d'être ceci ou cela et dans ce cas il n'a que l'identité vide de celui qui peut réaliser tous les possibles, de celui en qui tout est en puissance et rien en acte. Dès lors, dans le christianisme le témoignage manifeste un double aspect lié au caractère paradoxal de la vérité : le christianisme est d'une part un acte objectif qui a lieu dans le monde et d'autre part il est l'acte d'accueillir en soi, d'intérioriser un tel événement: «De là le sens profond du terme 'témoin' dans le christianisme; ce n'est ni un premier inventeur ni un réformateur de données existantes, mais un témoin, pour autant que le christianisme est un acte objectif qui se réalise dans le monde et pour autant que le témoin se l'assimile ${ }^{31}$. La révélation historique de ce qui n'est pas historique n'est pas rien et c'est cela qui donne au témoignage son importance dans la recherche de la vérité, mais le témoignage ne peut se réduire à la contingence du «j’y étais » et de ce point de vue le simple témoin oculaire n'est pas nécessairement le témoin du Christ. Certes, le témoin oculaire peut être un authentique témoin de l'Esprit, mais il n'est en rien avantagé par rapport aux hommes des générations futures : celui qui a entendu, vu, touché de ses mains peut n'avoir eu qu'un rapport tout à fait extérieur et en ce cas il n'est pas un témoin. Cela est particulièrement clair avec le Christianisme puisque la gloire du Christ échappe aux yeux de la chair et le simple contemporain peut l'avoir regardé sans l'avoir jamais $v^{32}$. Le témoin n'est pas ici le simple contemporain d'un fait contingent, mais le contemporain d'un fait absolu où l'éternel est devenu historique et qui n'est pas un passé, mais l'éternelle source de tout avenir. La vie du Christ est pour le chrétien le présent à partir duquel s'ouvre tout projet. Kierkegaard

\footnotetext{
27 Homélies sur l'Evangile de saint Jean, trad. franç. Marie-François Berrouard, BA 74b, Institut d'études augustiniennes, 1998, p.225.

28 A partir du II siècle se développe l'idée du «témoin de sang » qui peut aller jusqu'à la mort, mais il n'a pas plus de valeur que le témoin qui affronte chaque jour les violences du monde.

${ }_{29}$ Vingt et un articles de Faedrelandet, XIX, p.28.

${ }^{30}$ L'école du christianisme, XVII, p.182.

${ }^{31}$ Journal, II A452, tome 1, p.162.

32 Cf. Miettes philosophiques, VII, p.62.
} 
précise : «mais qu'est-ce à dire, sinon qu'on peut être contemporain sans l'être pourtant ; que l'on peut être contemporain et que, tout en exploitant cet avantage (au sens immédiat), on peut cependant être non-contemporain ; qu'est-ce à dire, sinon qu'il est impossible d'être immédiatement contemporain d'un tel maittre et d'un tel événement, de sorte que le contemporain réel ne l'est pas par suite de la contemporanéité immédiate, mais par suite d'autre chose $»^{33}$. On comprend alors que pour témoigner de la vérité la proximité historique a peu d'importance et qu'elle ne peut être au mieux que la cause occasionnelle de la contemporanéité réelle ${ }^{34}$. De même, le récit historique ne peut aussi qu'être l'occasion d'une contemporanéité réelle pour les hommes des générations futures. Etre témoin de la vérité n'a donc rien d'accidentel sans provenir pour autant d'une simple affirmation de soi puisque c'est toujours la vérité qui fait de moi un témoin. En cela, le témoin réel n'est pas celui qui sait se rendre immédiatement maitre d'un événement imprévu, mais il est celui qui sait se vider de lui-même pour laisser advenir en lui la vérité qui se manifeste. En conséquence, le témoin ne fixe pas de conditions à la vérité, ne serait-ce même que la capacité de pâtir, mais il s'efforce de répondre à ce que la vérité exige de lui. C'est la vérité qui fait le témoin et non pas le témoin la vérité et lorsqu'on oublie cela on retire inévitablement sa signification propre au témoignage en le réduisant à un simple mode de transmission d'une information. Il en va bien évidemment également de l'essence de la vérité : une vérité éthique qui aurait besoin d'être certifiée ne serait pas une vérité, tout comme prouver la vérité éthique du christianisme conduit nécessairement à le perdre comme vérité présente ${ }^{35}$.

Cependant, le témoin de la vérité n'est pas seulement celui qui s'abstient du vacarme du monde pour entendre une parole qui traverse les siècles, il est aussi le chevalier de la foi comme être paradoxal dont l'intériorité en est à son extrême degré de tension, tel Abraham qui dans son obéissance à Dieu comprend que tout bien vient de Dieu. Ce chevalier de la foi vit dans l'isolement absolu du témoin, isolement qui est diamétralement opposé à l'enfermement en soi du démoniaque : «Le vrai chevalier de la foi est un témoin, jamais un maitre; en cela réside sa profonde humanité autrement significative que cette frivole participation au bonheur et au malheur d'autrui, honorée sous le nom de sympathie et qui est pure vanité. Veut-on être simplement témoin : on confesse par là que nul, pas même le dernier des hommes, n'a besoin de compassion humaine, ou ne doit y trouver son avilissement pour qu'un autre s'en fasse un piédestal $»^{36}$. Si la pitié humaine n'est qu'une jouissance de soi dans laquelle se mélangent le mépris secret pour ceux que nous aidons et la recherche de leur admiration, non seulement elle est un abîme plus grand que l'indifférence à autrui, mais en outre en elle je me constitue comme spectateur de la souffrance d'autrui et je n'y suis pas constitué comme témoin. Contrairement à cela le chevalier de la foi n'est pas celui qui cherche à faire certifier son activité par autrui, ni un guide qui prend sa force de ceux qu'il aide, mais seulement un témoin capable de sortir de lui-même pour s'ouvrir à l'autre dans l'amour du prochain. Autrement dit, le témoin dans son isolement infini peut seul respecter la grandeur d'autrui, peut seul se sentir en dette infinie vis-à-vis de lui. Dès lors, la pleine signification du témoin ne se comprend vraiment qu'à partir de l'amour parce qu'en lui je suis saisi par l'excès de la vérité d'autrui et j'éprouve que j'ai contracté une dette infinie ${ }^{37}$. Est au plus haut point témoin celui qui aime parce qu'il ne peut pas prouver son amour, parce que toute preuve serait indigne de cet amour, mais qu'il peut justement seulement en témoigner et que ce témoignage consiste à dire sa dette infinie: dans l'amour je me reçois de celui qui me donne d'être, de celui qui en se

\footnotetext{
33 Ibidem, p.62-63.

34 Cf. ibidem, p.97.

${ }^{35}$ Cf. Post-scriptum, X, p.30.

${ }^{36}$ Crainte et tremblement, V, p.170.

${ }^{37}$ Cf. Les auvres de l'amour, XIV, p.161.
} 
laissant aimer me donne de me révéler. Le témoin n'est donc ni le juge d'autrui, ni le juge de lui-même, ni le juge de son amour, mais il est celui qui éprouve ce qui est exigé de lui dans cet amour, qui éprouve cette vérité qu'il doit demeurer en dette d'amour. En conséquence, seul le témoin peut prêcher véritablement le christianisme en étant en dette d'amour : "Qu'est-ce qu'un témoin? C'est quelqu'un qui immédiatement fournit la preuve de la doctrine qu'il prêche - je dis immédiatement et parce que c'est la vérité en lui et béatitude, et parce qu'à l'instant il offre sa personne et dit: voyez si vous pouvez me contraindre à renier cette mienne doctrine $»^{38}$.

De ce point de vue, Kierkegaard s'oppose délibérément à la conception hégélienne du témoignage ${ }^{39}$. Certes, pour Hegel, dans la foi mon esprit rend témoignage à la vérité : «Nous pouvons définir la foi véritable ainsi : elle est le témoignage de mon esprit, le témoignage de l'esprit au sujet de l'esprit ${ }^{40}$. Cela signifie bien que dans le témoignage il n'y a de place pour aucun contenu extérieur et que cette représentation n'a pas encore pour autant la nécessité du concept. En effet, selon Hegel, la religion chrétienne est d'abord une doctrine et dès lors la vraie foi ne peut avoir aucun contenu contingent : « La foi repose sur le témoignage, elle a ainsi un fondement. Mais le fondement absolu proprement dit de la foi, le témoignage absolu concernant le contenu d'une religion est le témoignage de l'esprit, non pas des miracles, non pas une accréditation extérieure, historique $»^{41}$. En fonction de sa thèse directrice, selon laquelle le plus intérieur est le plus spéculatif, Hegel ne peut comprendre le témoignage que d'une façon négative, c'est-à-dire comme une simple adhésion intérieure, comme une sympathie accompagnée de discernement et de culture, qui doit cependant être dépassée dans la mesure où le véritable témoignage de l'esprit n'a plus aucun élément positif, comme le miracle, ni d'élément subjectif, comme la sympathie, et s'identifie alors à la philosophie elle-même où le concept tire de son propre fond la vérité. Pour Kierkegaard au contraire, le caractère historique et extérieur du témoignage n'est pas contingent : le témoin de la vérité n'est pas celui qui accède à la nécessité du concept, mais celui qui éprouve sa contemporanéité avec un événement historique, unique, absolument singulier et radicalement nouveau. La condition de possibilité du témoignage n'est pas ici une qualité du sujet, mais une décision libre de Dieu qui fait époque et appelle dans l'histoire ma propre résolution. En effet, le pouvoir de témoigner de la vérité ne peut être une capacité a priori du sujet et l'intériorité du témoignage est un recueillement dans lequel se brise la simple vie auprès de soi : elle est un mouvement, un désir, issu de «l'objet » luimême qui appelle à témoigner. Certes, parler de «l'objet» du témoignage est déjà un abus de langage dans la mesure où ce dont je témoigne n'est justement pas encore constitué en objet identifiable, mais est ce qui me saisit depuis un ailleurs et ainsi toujours me surprend en me faisant être là où je ne m'imaginais pas pouvoir être. Le témoin ne commence donc pas par cet acte de possession qui consiste à thématiser en objet ce qui arrive. En effet, il est tout entier dans l'acte d'accueillir, c'est-à-dire de vivre le moment apparitionnel du «il y a ", de vivre la surprise d'être là où le réel me saisit. En cela, le témoin est prêt à tout et débordé par tout, il est ouvert à l'altérité du réel sans projet, ni souci. Ainsi, contre Hegel, on peut reconnaître avec Kierkegaard que le témoignage est en lui-même une sortie d'Egypte, de tout ce qui me retient prisonnier en moi-même, parce qu'il n'y a de témoignage que d'un événement, c'est-à-dire de ce qui n'est jamais objet, de ce qui ne peut jamais être mis à découvert. En effet, l'événement est ce que je ne peux produire, inventer ou anticiper, mais qui toujours surgit de l'imprévisible et qui marque, pour reprendre une

\footnotetext{
${ }^{38}$ Journal, X3 A6, tome 4, p.8-9.

39 Pour le contexte théologique, cf. Jean-Yves Lacoste, "Kierkegaard face aux théologies de son temps », revue Oblique "Kierkegaard », p.103-107, Paris, 1981.

${ }^{40}$ Leçons sur la philosophie de la religion, ${ }^{\text {ère }}$ partie, trad. franç. P. Garniron, PUF, Epiméthée, 1996, p.223.

${ }^{41}$ Ibidem, p.268.
} 
catégorie d'Henri Maldiney, la transpassibilité de notre existence ${ }^{42}$. Ainsi, le témoignage est existence parce qu'en lui le sujet ne se contente pas de persister dans l'être en se constituant lui-même, mais au contraire il devient autre, il sort de lui-même, de par la force de ce qui le surprend : il est là où l'événement l'appelle et dans cette sorte d'intentionnalité inversée il reçoit de ce qui le saisit son vrai visage en recevant de ce qui n'est pas lui le pouvoir de se possibiliser. Le témoin de la vérité ne peut donc plus être compris comme une intériorité se libérant de toute altération par une extériorité de façon à n'être plus que le pur témoignage de l'esprit par l'esprit. Kierkegaard permet en cela de rompre avec la représentation d'un sujet-substance demeurant identique à lui-même dans l'abri de sa transcendance et qui dans cette simple persistance vide se croit libre seulement parce qu'il n'est pas modifié par ce qui lui arrive. Il est essentiel de déconstruire cette évidence de la présence à soi, sans doute plus difficile à remettre en cause que l'évidence de la conception galiléenne de la nature, parce qu'elle en est l'origine, pour pouvoir se comprendre comme témoin, c'est-à-dire comme un être qui se fait donner par ce qui lui advient le pouvoir de recommencer comme un autre, le pouvoir de se temporaliser. Pour Kierkegaard le témoignage s'inscrit ainsi dans une détermination du temps comme espérance puisque la vérité dont on a à témoigner de façon insubstituable est la source de toute possibilisation, c'est-à-dire de toute vie. En me révélant l'unicité absolue de mon existence, et en me libérant d'une identité pensée comme la simple persistance d'une essence, le témoignage se donne lui-même comme irréductible au savoir absolu et il souligne le caractère nécessairement paradoxal de la vérité : il rapporte en effet comment la vérité s'est manifestée dans l'histoire et en quoi elle est une synthèse de contingence et de nécessité dans la mesure où le plus contingent se révèle ici comme le plus nécessaire ${ }^{43}$.

Témoigner de la vérité c'est donc devenir ce que l'on est, mais que l'on n'imaginait pas être, en étant libéré de l'enfermement en soi par la vérité elle-même qui n'est jamais objet mais toujours événement. Le plus haut degré de l'intériorité consiste à recevoir ces paroles de vérité comme si elles étaient siennes, mais cette possibilité et cette liberté viennent, pour Kierkegaard, de Dieu lui-même qui donne de l'indépendance vis-à-vis de lui ${ }^{44}$ et témoigner c'est alors transmettre ce don de la liberté à chacun de ses semblables. En me rendant ainsi libre la vérité fait de moi une personne, c'est-à-dire non pas cet être abstrait pour lequel tout est possible et qui erre dans le monde en cherchant à combler son propre vide, mais cet être qui est «une synthèse de possibilité et de nécessité »" ${ }^{45}$ Le témoignage est précisément cette respiration qui me fait vivre, c'est-à-dire qui m'ouvre à mes possibles et donc forme ma personnalité. En effet, la personnalité est ce qu'on ne peut pas perdre sans se perdre soi-même ${ }^{46}$, mais elle ne se comprend plus cette fois comme la vide conscience de soi puisqu'elle est au contraire cet accueil de l'événement de la vérité, accueil sans lequel le moi est perdu et sans lequel l'homme tombe dans le désespoir du fatalisme. Le témoin de la vérité sait lui que tout est possible à Dieu : tel est le saut de la foi, la sortie d'Egypte qui fonde toute espérance. La personne est ici l'existant qui témoigne de la vérité qui lui donne d'être une vie toujours en commencement, toujours renaissante.

42 Henri Maldiney n'a cessé de méditer cette idée que le réel est ce que l'on n'attendait pas et la phénoménologie contemporaine de l'événement devra un jour reconnaître sa dette envers les analyses d'Henri Maldiney si elle ne veut pas se perdre dans une répétition somnambulique.

43 En élucidant la vérité comme paradoxe et donc comme ce que je ne produis pas et ne possède pas, Kierkegaard, contrairement à ce que dit Heidegger (GA 17, Einführung in die phänomenologische Forschung, Klostermann, 1994, p.126: "Auch des 'Paradox' bei Kierkegaard ist nicht anders als das Resultat des fundamentalen Versäumnisses der Interpretation der Idee der Wabrheit »), n'a pas « raté »l'élucidation d'un nouveau sens de l'idée de vérité. La compréhension de la vérité comme paradoxe ne reconduit-elle pas plus sûrement à la vie même de la personne que la compréhension existentiale de la vérité qui continue à faire de la vie une possibilité du Dasein?

${ }^{44}$ Cf. Post-scriptum, X, p.241.

${ }^{45}$ La maladie à la mort, XVI, p.197.

${ }^{46}$ Cf. Jacques Colette, Histoire et absolu, Desclée, 1972, p.254. 
Ainsi, la personne est témoignage parce que la vérité elle-même est une personne et qu'on ne peut être soi que dans ce oui à la vérité qui se donne et qui en se donnant nous donne d'être libre. Il ne s'agit plus ici de choisir entre la vérité et le mensonge parce qu'il n'y a pas de lieu neutre d'où un tel choix pourrait avoir lieu: on est toujours déjà ou dans le témoignage de la vérité ou dans la haine de la vérité. En effet, pour dire oui ou pour dire non à la vérité il faut qu'elle soit donnée et en cela le témoin de la vérité ne peut jamais faire de la vérité son objet, mais il ne peut que la recevoir comme un pur don relativement au Christ comme oui de Dieu, comme oui de celui qui s'est totalement donné sur la croix. Le oui du témoignage humain ne peut être séparé pour Kierkegaard du oui du témoin fidèle qui est ainsi l'unité de tous les témoignages humains qui font vivre notre alliance à la vérité.

\section{Le martyre ou la vérité en souffrance}

On ne témoigne de la vérité qu'en se faisant le contemporain de son événement et c'est cet acquiescement à la vérité qui disperse la foule parce qu'il est une réponse à ce que la sagesse du monde ne peut pas anticiper, préparer. En effet, dans ce oui au don de la vérité, l'homme risque sa vie en s'arrachant à la temporalité de la répétition et ce risque est ce que la sagacité et la médiocrité de ce monde veulent justement éviter. En cela l'instant du témoignage n'est dépendant d'aucune condition mondaine, comme le dit Kierkegaard dans une très belle page de 1855 : "Mais quand paraît l'homme de la situation, l'instant est là. Car l'instant, c'est justement ce qui n'est pas dans les circonstances ${ }^{47}$. De ce point de vue, pour Kierkegaard, «la foi seule est la possibilité de l'instant ${ }^{48}$ en ce qu'elle est une fidélité à ce qui est au-delà de toute attente. Or, cette possibilité est d'abord la possibilité de différer de soi et c'est pourquoi la nature propre du témoignage ne peut se comprendre qu'à partir de l'élucidation du lien essentiel entre devenir soi et souffrance puisqu'il s'agit ici d'une souffrance qui n'est pas dépendante des circonstances extérieures de l'existence. La souffrance qui est la marque de l'authenticité humaine est la souffrance de ne pas être soi parce qu'on ne peut être vrai que par une vérité qui ne vient pas de soi. En cela le martyre est le mode d'être de l'homme comme être créé à l'image de Dieu : il concentre en lui ce qui fait notre humanité c'est-à-dire le caractère paradoxal de notre existence.

Dès que le chrétien témoigne pour la vérité la souffrance n'est pas en reste ${ }^{49}$ dans la mesure où ce témoignage est incompatible avec le monde. Il appartient donc à l'essence du témoin de souffrir pour la vérité et Kierkegaard précise le sens de cette souffrance dans un texte de 1854 où il se demande si l'évêque Mynster était un vrai témoin de la vérité : «Un témoin de la vérité, c'est un homme dont la vie ignore, du commencement à la fin, tout ce qu'on appelle jouissance - et que peu ou beaucoup te soit accordé, tu sais combien on aime ce qu'on appelle jouissance; mais sa vie a ignoré du commencement à la fin ce qu'on appelle ainsi ; en revanche, du commencement a la fin, elle a été vouée à tout ce qu'on appelle souffrance $»^{50}$. Dès lors, ne peut parvenir au soi authentique que celui qui s'est arraché au désespoir de ne pas être soi dans lequel maintient la seule jouissance de soi. Pour expliciter cela Kierkegaard distingue plusieurs formes de cette souffrance et elle est d'abord une souffrance assez connue : «Un témoin de la vérité, c'est un homme qui témoigne pour la vérité dans la pauvreté, la médiocrité de condition et l'abaissement etc. $»^{51}$. Cependant, cette souffrance reconduit à une souffrance plus intérieure : "Sa vie a été vouée aux luttes intérieures, à la crainte et au tremblement, au frémissement, aux tentations spirituelles, aux angoisses de l'âme, aux tourments de l'esprit $»^{52}$. Cela signifie bien que cette souffrance

\footnotetext{
${ }^{47}$ L'instant, XIX, p.298.

${ }^{48}$ Ibidem, p. 299.

${ }^{49}$ Cf. Journal, X3 A272, tome 4, p.85.

${ }^{50}$ Vingt et un article de Faedrelandet, XIX, p.6.

${ }^{51}$ Ibidem.

52 Ibidem.
} 
n'est ni seulement initiale, ni contingente, mais qu'elle est liée à l'acte même de témoigner dont la possibilité reconduit à la grâce divine qui peut seule permettre de supporter cette souffrance. Le témoin de la vérité n'est alors pas celui qui demande à être soulagé de cette souffrance, mais celui qui demande à Dieu de le fortifier pour qu'il rentre de plus en plus dans cette souffrance et donc pour que le désir de Dieu s'inscrive en lui d'une façon de plus en plus profonde: "Mais celle (la consolation) du témoin de la vérité, c'est que Dieu le fortifie pour tenir bon dans la souffrance, sa prière l'enfonce donc de plus en plus profond dans la souffrance, plus elle a de ferveur et plus il est proche de Dieu, plus il se fixe dans la souffrance : as-tu le courage de prier ainsi ? ${ }^{53}$ ” Il est donc impossible de témoigner pour la vérité sans exposer sa vie elle-même parce qu’il y a hétérogénéité entre cette vérité et le monde, y compris avec cette part de soi qui appartient au monde. Tout vrai chrétien est en cela un martyr qui ne se contente pas de tirer profit d'une doctrine. En effet, en lui-même le témoin n'a aucune habileté, il n'a aucun pouvoir au sens du monde, et sa seule autorité vient de la vérité elle-même qu'il transmet et c'est pourquoi non seulement il est hétérogène au monde, mais qu'il se doit aussi d'aller dans le monde pour le transformer. Ainsi, parce qu'il reçoit la possibilité de transformer le monde, c'est-à-dire de la saisir à partir d'un ailleurs, le témoin de la vérité n'est pas là pour jouir de lui-même, mais pour souffrir et c'est pourquoi il ne doit pas chercher la quiétude dans ses rapports avec le monde, mais la violence qui consiste justement à témoigner, à dire au monde une vérité qui n'est pas du monde.

Témoigner de la vérité n'est-ce pas alors au-delà des possibilités de l'homme ? A cette question Kierkegaard a déjà répondu que le témoignage ne peut être compris comme une capacité a priori du sujet et qu'il relève d'une ipséité par grâce, mais il précise en outre que dans ce domaine le Christ ne doit pas être considéré comme un modèle. Alors que pour beaucoup d'éthiques le Christ demeure un idéal à atteindre, Kierkegaard souligne que si le Christ est mort pour nous sauver c'est pour que nous n'ayons pas à mourir comme lui et dans cette perspective on peut considérer que seul l'homme est un témoin de la vérité. En effet, dans le témoignage il ne s'agit pas de vouloir mourir en faisant de sa mort l'ultime lieu d'une affirmation de soi, d'une jouissance de soi, mais bien de vivre en aimant la vérité plus que soi-même, de vivre dans l'amour sans limite de ce qui nous donne d'être. Le chemin vers soi passe par le monde et il s'agit toute sa vie de mourir au monde pour mieux le transformer et ce martyr est plus difficile que la mort elle-même ${ }^{54}$. Il ne s'agit donc pas tant de perdre la vie, ce qui peut déjà avoir lieu en cette vie dans le démoniaque qui ferme à toute possibilité, que de la donner et chaque homme en cela doit découvrir sa façon propre d'être un témoin de la vérité : "Mais dans la réflexion, ce qui exprime la plus grande ressemblance possible avec l'homme-Dieu, c'est précisément d'avouer qu'aucun homme n'a le droit de se faire tuer $\aleph^{55}$. Autrement dit, le témoin de la vérité demeure un homme parmi les hommes, non le juge des autres hommes, et si «c'est de martyrs qu'on a besoin et pas d'autre chose $»^{56}$, c'est parce qu'ils invitent à la vigilance, nous qui avons toujours tendance à nous enfermer dans une quiétude où aucune vérité ne se révèle. La souffrance prise en ce sens est notre pouvoir-être, notre pouvoir de nous révéler en différant de nousmêmes : on ne peut trouver notre ipséité, notre consolation et notre espérance qu'en elle. Dès lors, si dans cette épreuve l'homme décide de sa vérité, l'humilité accompagne aussi le martyr qui ne peut se prendre pour un modèle, qui ne peut jamais dire «je suis la vérité ». En effet, l'humilité est la sœur de la souffrance dans la mesure où seule la souffrance enseigne à être ce que l'on pense et ce que l'on dit et où seul celui qui fait en elle l'épreuve de ce qu'il en coûte d'être soi peut renoncer à vouloir posséder la vérité. Abraham est en

\footnotetext{
53 Journal, X4 A565, tome 4, p.337.

${ }^{54}$ Cf. David Brézis, Temps et présence, Vrin, 1991, p.168.

55 Journal, X1 A328, tome 3, p.108.

${ }_{56}$ Journal, X1 A16, tome 3, p.16.
} 
cela le vrai témoin, victorieux d'une tentation divine, parce qu'il témoigne par sa foi non de l'homme et de son courage, mais bien de Dieu. La vie humaine peut ainsi trouver son unité dans cette passion de la foi, dans ce devoir absolu d'aimer Dieu qui est un abandon absolu. De ce fait, loin de toute neutralité, de toute extériorité, témoigner de la vérité est une lutte de laquelle nul ne peut sortir indemne. Ne faut-il pas alors reconnaitre dans la souffrance une réduction phénoménologique dans laquelle je ne pers rien de ce que je suis, mais où tout ce qui me constitue ne paraît plus centré autour de la persistance de la jouissance de soi. En effet, la souffrance destitue le je et sa prétention à la neutralité pour me faire comprendre que je suis là où le devoir absolu m'appelle puisque je ne suis pas la condition d'un tel devoir, mais que c'est ce devoir d'aimer Dieu qui est la condition de mon être. Dans le témoignage, je suis d'abord dessaisi de ma parole, silencieux, et c'est ensuite que la vérité de ma parole, sa véracité, tient à ce fait d'être dessaisi par la vérité dont je témoigne. Celui qui souffre pour la vérité, qui est devenu entièrement faible dans cette passion, est aussi devenu fort par cette vérité parce qu'alors, et dans un sens propre, il ne peut être autrement qu'il n'est dans son abandon à l'amour de la vérité. Dès lors, la philosophie du témoignage ne relève pas d'une philosophie de l'interprétation, mais d'abord et avant tout d'une métaphysique de la subjectivité pour laquelle le soi religieux est celui pour lequel le témoignage est en lui-même attestation, c'est-à-dire ouverture à ses propres possibilités. En effet, ma certitude d'exister sur le mode de l'ipséité ne me vient pas de moi, mais de ce dont je témoigne. On peut alors comprendre en quoi ce n'est jamais le je qui témoigne puisque le témoin n'est pas seulement celui qui souffre pour la vérité, mais plus essentiellement il est celui en qui la vérité elle-même est en souffrance, celui qui précisément laisse le Christ, le témoin fidèle, témoigner en lui. Le martyr, qui peut prendre des formes très diverses, consiste toujours à laisser témoigner en soi celui qui a révélé l'amour du Père et ainsi à laisser à l'amour divin son espace de révélation. Le témoin de la vérité est la personne qui trouve sa consolation dans le fait de pouvoir souffrir pour la vérité parce que la vérité ellemême est une personne en souffrance en ce monde. Ainsi le martyr, en souffrant dans le monde, découvre que la plus grande passivité est la plus grande intériorité, celle qui ne trouve jamais la paix et la satisfaction et celle qui creuse au fond d'elle-même, seule avec Dieu, sa responsabilité devant Dieu, celle qui approfondit sans cesse la souffrance de la responsabilité. Même si cette solitude du chevalier de la foi est problématique, même si Kierkegaard n'envisage pas vraiment la communauté du témoignage, il n'en demeure pas moins qu'il nous donne à comprendre le témoignage comme une prière qui répond à un appel, c'est-à-dire comme une respiration qui sans cesse me renouvelle en m'appelant à être ce que je ne suis pas et en ouvrant ainsi le moi à la possibilité qui est son oxygène ${ }^{57}$. Témoigner revient à prier, à respirer et à être ainsi renouvelé par la vérité et c'est cela devenir soi.

\section{Conclusion : du je au témoin}

Kierkegaard s'est proposé de donner à voir le vrai sens du concept biblique de témoignage, mais en décrivant le témoignage comme le mode d'être de l'homme exposé à la vérité, il permet aussi de remettre en cause toute scission du concept de témoignage (juridique, historique, biblique, philosophique, etc.) qui fait nécessairement perdre le concept lui-même. En effet, il n'est pas question pour Kierkegaard d'étudier le témoignage biblique, pour ensuite construire un concept philosophique de témoignage, en réduisant tout ce qui est religieux, puisqu'une telle méthode, au-delà de son arbitraire, ne peut qu'isoler la réflexion sur le témoignage d'une réflexion sur la vérité. La conséquence de cet isolement est soit de faire du témoignage une simple enveloppe vide, soit de le mettre en dépendance d'une détermination impensée de la vérité. En cela Kierkegaard est l'un des premiers à nous libérer d'un concept vulgaire de témoin qui le réduit à n'être qu'un simple

${ }^{57}$ Cf. La maladie à la mort, XVI, p.197-198 et Journal, IX A462, tome 2, p.384. 
observateur actif et critique de ce qui a lieu. Un tel renversement n'a été possible qu'à partir d'une compréhension de notre essentielle historicité et de notre nécessaire exposition à la vérité. Alors que le concept vulgaire de témoin fait de lui ce qui est en puissance de toute chose, y compris de Dieu, mais en acte de rien, puisqu'il fixe a priori les conditions de tout avoir lieu, le vrai témoin à la fois est une synthèse de nécessité et de possibilité et d'une certaine façon n'a pas de limite, pas de limite qui viendrait d'un je. En effet, même comme possibilité pure le je doit bien dire jusque là c'est moi, ensuite ce n'est plus moi, puisqu'il n'est qu'un observateur pour lequel ce qui a lieu est un en-face, qui est par lui-même et qui advient ensuite à l'homme qui le constitue en objet à partir de son regard critique, alors que le témoin, lui, ne vit pas dans un tel espace de représentation limité, mais dans un espace de présence où l'inimaginable est possible. Dès lors, en comprenant la personne comme paradoxe, Kierkegaard ouvre, même s'il n'est pas nécessairement sur ce point le premier, à une intelligence existentielle de la vérité dans laquelle le témoignage se trouve délivré de toute relativité pour être le mode même de notre rapport à l'absolu. En cela je ne peux devenir un témoin, c'est-à-dire entrer dans un rapport absolu à l'absolu, que parce que la vérité me précède et me saisit au cœur de ma non-vérité. C’est donc la vérité elle-même comme événement qui rend ontologiquement possible le témoin qui est bien, comme le dira Heidegger, «dans » la vérité dont il parle. Un tel être dans la vérité est la véritable intériorité existentielle, qui se distingue radicalement de l'intériorité spéculative, qui ne peut qu'enfermer dans le désespoir parce qu'elle ne peut ouvrir à ma possibilité.

Ainsi, la souffrance en opérant une réduction phénoménologique rend aussi possible une eidétique du témoignage qui dévoile le témoin comme n'étant ni un spectateur, ni un propriétaire, ni un conservateur, ni un producteur, ni un juge, ni un maître qui argumente, mais comme étant celui qui véritablement aime la vérité qui se donne lui au point de renoncer à la jouissance de soi pour tenter d'être cette vérité et d'en donner ainsi une preuve pratique. Autrement dit, il n'y a de témoignage de la vérité que quand on ne réduit pas ce témoignage à un témoignage de soi. En retour, en témoignant de cette vérité qui nous excède, elle nous révèle aussi dans un pouvoir être inimaginable a priori. Si le témoignage est communication, il est d'abord la communication directe-indirecte d'un devoir-être qui appelle à donner sa vie par amour. Cependant, si dans cette communication le témoin parle en première personne, c'est la vérité elle-même qui le constitue comme tel. Dès lors, il faut bien reconnaître avec Jean-Luc Marion que «sous le titre de témoin, il faut entendre la subjectivité dépouillée des caractères qui lui donnaient un rang transcendantal $\aleph^{58}$. En effet, le je deviens témoin par cette inversion du principe de l'intériorité qui ne fait plus d'elle la région originaire où se constituent tous les objets, et dans laquelle je reconnais ma passivité radicale devant l'excès de ce qui se manifeste à moi. En cela le témoin éprouve son impuissance radicale à prendre de la distance, de la hauteur, pour s'éprouver lui-même dans une pure identité hors du monde et ensuite constituer et co-constituer en objet tout ce qui se donne à lui ${ }^{59}$. Ne faut-il pas alors reconnaitre que ce n'est pas seulement par notre regard que nous sommes témoins, mais par notre corps tout entier? Quoi qu'il en soit, une telle substitution du témoin au je ne peut être attribuée au seul caractère théologique de l'œuvre de Kierkegaard dans la mesure même où elle dévoile le caractère essentiel de la vérité comme intériorité, de la vérité en tant qu'elle me concerne, en tant qu'il n'est pas indifférent qu'elle se donne à moi. Ainsi, parce que la vérité m'édifie, la question essentielle n'est pas pour Kierkegaard de savoir si je suis, mais de savoir à quoi je suis appelé. Seule cette question m’importe parce que seule elle fait de moi une question

${ }^{58}$ Etant donné, PUF, 1997, p.302.

59 Jean-Luc Marion écrit encore, synthétisant ainsi en partie le projet d'Etant donné: "Le témoin s'oppose donc au Je en ce qu'il n'a plus l'initiative de la manifestation (par facticité), ne voit pas en totalité le phénomène donné (par excès d'intuition), ne peut lire ni interpréter l'excès d'intuition (par pénurie de concepts) et enfin se laisse juger (dire, déterminer) par ce qu'il ne peu lui-même dire, ni penser adéquatement »(p.303). 
alors que la vérité objective produite par un je ne met pas ce je lui-même en question parce qu'elle ne me concerne pas, parce que je n'ai pas à être ce que je produis. Le tremblement, impensable pour un je, est la marque d'une vérité qui ne se montre qu'en m'appelant à être, c'est-à-dire à renaître. En un sens, toute vérité édifie, même si elle n'est pas chrétienne, parce qu'elle est une assignation à témoigner, c'est-à-dire à répondre depuis sa non-vérité à l'appel de l'être pour montrer ce que peut cette vérité qui m'excède et me constitue. En conséquence, le témoin étant celui qui se reçoit de ce qui lui advient, la non-vérité de l'existant, plus originelle que l'homme, destitue plus radicalement et plus sûrement le je que toute pensée de la finitude dans laquelle la vérité demeure relative à l'existant. Ainsi, Kierkegaard, en écrivant, dans Coupable ?-Non coupable?, "Le religieux m'intéresse comme phénomène, et comme le phénomène qui m’intéresse le plus $»^{60}$, indique que c'est bien à partir de ce phénomène que je peux prendre conscience de la possibilité de m'ouvrir à la vérité seulement là où je ne me prends pas pour elle, seulement là où je saisis le caractère incommensurable de son extériorité vis-à-vis de mon intériorité. En effet, c'est seulement à partir de ce phénomène que je peux comprendre ce que signifie devenir un témoin, c'est-àdire l'Individu qui effectue ce saut, incompréhensible pour les autres hommes, dans lequel il sait qu'il n'est que parce que la vérité est et qui ainsi vit, tel Abraham, dans l'abandon le plus total à la vérité. L'oubli de ce phénomène religieux n'est-il pas aussi un «ratage » dans l'interprétation de l'idée de vérité qui interdit de penser la vérité autrement que comme relative à autre chose qu'elle et qui interdit donc aussi de reconnaître l'essence authentique du témoin pour lequel la vérité est le chemin et la vie ?

${ }^{60} \mathrm{IX}$, p.426. 